\title{
Steady Fock states via atomic reservoir
}

\author{
F. O. Prado ${ }^{1}$, W. Rosado ${ }^{2}$, G. D. de Moraes Neto ${ }^{2}$, and M. H. Y. Moussa ${ }^{2}$ \\ ${ }^{1}$ Universidade Federal do ABC, Santo André, São Paulo, Brazil and \\ ${ }^{2}$ Instituto de Física de São Carlos, Universidade de São Paulo, São Carlos, São Paulo, Brazil
}

In this letter we present a strategy that combines the action of cavity damping mechanisms with that of an engineered atomic reservoir to drive an initial thermal distribution to a Fock equilibrium state. The same technique can be used to slice probability distributions in the Fock space, thus allowing the preparation of a variety of nonclassical equilibrium states.

PACS numbers: 32.80.-t, 42.50.Ct, 42.50.Dv

The development of strategies to prepare nonclassical states [1] and, in particular, to circumvent their decoherence — via decoherence-free subspaces [2], dynamical decoupling [3], and reservoir engineering [4, 5] - have long played a significant role in quantum optics. On the conceptual side, the need for these states stems from their use in the study of fundamental quantum processes, such as decoherence [6] and the quantum to classical transition [7]. On the pragmatic side, the advent of quantum computation and communication - which depends strongly on successfully producing highly nonclassical states and ensuring their long-term coherence [8] - has certainly put extra pressure on researchers to implement efficient techniques of engineering and protection of nonclassical states. The proposition of schemes that enable the generation of nonclassical equilibrium states thus represents an ideal approach to the current challenges. In this regard, the reservoir engineering technique proposed in Ref. 4] and experimentally demonstrated in a trapped ion system [9] signals an important step toward the implementation of quantum information processes [8], a goal that has recently mobilized practically all areas of low-energy physics. Reservoir engineering, however, has major limitations, starting with the fact that it prevents, for example, the generation of Fock equilibrium states (a key goal of the present letter). Moreover, the protection of a particular state demands the (not-always-easy) engineering of a specific interaction which the system of interest is forced to perform with other auxiliary quantum systems. 
Recently, the generation of Fock states with photon numbers $n$ up to 7 was reported in cavity QED, where a quantum feedback procedure is employed to correct decoherenceinduced quantum jumps [10]. The resulting photon number distribution assigns a probability around 0.8 to the generation of number states up to 3 , falling to below 0.4 for $n=7$. Nonequilibrium number states up to 2 photons have long been prepared in cavity QED [11], as well as in most suitable platforms, such as ion traps [12] and, lately, in circuit QED [13], where number states up to 6 were achieved.

In this letter we present a protocol in which the atomic beam reservoir technique [14] is exploited to produce high fidelity equilibrium Fock states in cavity QED. The atomic reservoir - built up by injecting a beam of atoms that interact, one at a time, with the cavity mode - prompts the emergence of an engineered Liouvillian superoperator to govern the cavity field dynamics, alongside that coming from the cavity loss mechanisms. We stress, from a practical perspective, that atomic reservoirs have for some time been used for the preparation of the cavity vacuum state [15]. Moreover, this has been theoretically explored, in close relation to the reservoir engineering technique [4], for the generation of an EinsteinPodolsky-Rosen steady state comprising two squeezed modes of a high finesse cavity [16]. Our proposal, however, is altogether different from those in Ref. [4, 16]. In contrast to the assumptions that support the method proposed in [4, 16], our protected Fock state is not a steady state of a specific engineered Lindbladian $\mathcal{L} \rho=(\Gamma / 2)\left(2 \mathcal{O} \rho \mathcal{O}^{\dagger}-\mathcal{O}^{\dagger} \mathcal{O} \rho-\rho \mathcal{O}^{\dagger} \mathcal{O}\right)$, where the only pure steady state of the system is the eigenstate of operator $\mathcal{O}$ with a null eigenvalue. In our protocol, the steady state is driven by a sum of three engineered Lindbladians, two of which act on selected subspaces of the cavity mode space, the mode emitting or absorbing photons within these subspaces. The third Lindbladian is associated with (nonselective) photon absorption by the cavity mode, in order to counterbalance the inevitable emission to the natural (nonengineered) environment. The selective Lindbladians are built up from engineered selective Jaynes-Cummings (JC) Hamiltonians, while the Lindbladian for photon absorption follows from a usual JC interaction.

To generate the required Lindbladians, we rely on engineered selective Hamiltonians [17] and engineered atomic reservoirs [14, 16], the latter demanding a beam of atoms to cross a high-Q cavity. The selective Lindbladians are engineered by assuming the atomic level configuration in Fig. 1(a), the emission or absorption process following from the atoms prepared in the ground $|g\rangle$ or excited $|e\rangle$ state, respectively. The (non-selective) Lindbladian 
(a)

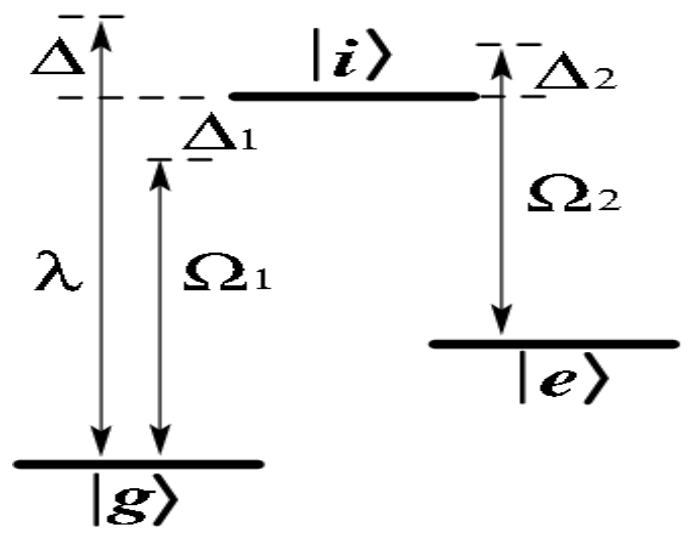

(b)

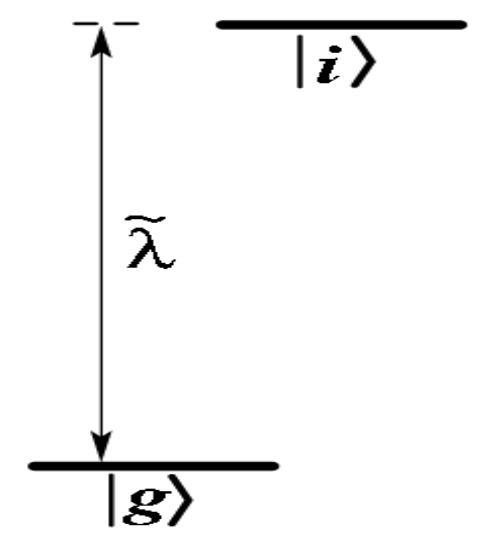

FIG. 1: Atomic level configurations to engineer (a) selective and (b) non-selective Hamiltonians.

accounting for photon absorption is engineered from the level configuration in Fig. 1(b). As shown in Fig. 1(a), the cavity mode $(\omega)$ is used to promote a Raman-type transition $g \leftrightarrow e$, helped by two laser beams, $\omega_{1}$ and $\omega_{2}$, out of tune with transitions $g \leftrightarrow i$ and $e \leftrightarrow i$, respectively. In the configuration in Fig 1(b) —which follows from that in Fig. 1(a) by taking advantage of the Stark effect and switching off the laser beams - the cavity mode is now used to couple resonantly the shifted levels $|g\rangle$ and $|i\rangle$. When the configuration is that in Fig 1(a), the atoms are randomly prepared in the states $|g\rangle$ and $|e\rangle$, and when that in Fig. 1(b), the atoms are prepared at the auxiliary level $|i\rangle$. Starting with the engineering of the selective JC interactions arising from the diagram in Fig. 1(a), we write the Hamiltonian

$$
H=\lambda \sigma_{i g} a \mathrm{e}^{-i \Delta t}+\Omega_{1} \sigma_{i g} \mathrm{e}^{i \Delta_{1} t}+\Omega_{2} \sigma_{i e} \mathrm{e}^{-i \Delta_{2} t}+H . c .,
$$

where $\sigma_{r s}=|r\rangle\langle s|, r$ and $s$ labelling the atomic states involved, and we define $\Delta=\omega-\omega_{i g}$, $\Delta_{1}=\omega_{i g}-\omega_{1}$, and $\Delta_{2}=\omega_{2}-\omega_{i e}$, with $\omega_{i \ell}=\omega_{i}-\omega_{\ell}(\ell=g, e)$. It is straightforward to verify that the conditions $\lambda \sqrt{n+1} \ll \Delta$ and $\Omega_{j} \ll \Delta_{j}(j=1,2)$ lead to the effective interaction $([18])$

$$
\begin{aligned}
H_{e f f} & =\left(\xi a^{\dagger} a-\varpi_{g}\right) \sigma_{g g}+\varpi_{e} \sigma_{e e} \\
& +\left(\zeta a^{\dagger} \mathrm{e}^{i \delta t} \sigma_{g e}+H . c .\right)
\end{aligned}
$$


where $\varpi_{g}=\left|\Omega_{1}\right|^{2} / \Delta_{1}$ and $\varpi_{e}=\left|\Omega_{2}\right|^{2} / \Delta_{2}$ stand for frequency level shifts due to the action of the classical fields, whereas the strengths $\xi=|\lambda|^{2} / \Delta$ and $\zeta=\lambda^{*} \Omega_{2}\left(\Delta^{-1}+\Delta_{2}^{-1}\right) / 2$ stand respectively for off- and on-resonant atom-field couplings to be used to engineer the required selective interactions; finally, $\delta=\Delta-\Delta_{2}$ refers to a convenient detuning to be specified in the following lines. To get selectivity, we first perform the unitary transformation $U=\exp \left\{-i\left[\left(\xi a^{\dagger} a+\varpi_{g}\right) \sigma_{g g}+\varpi_{e} \sigma_{e e}\right] t\right\}$, which takes $H_{e f f}$ into the form

$$
V_{e f f}=\sum_{n=1}^{\infty} \zeta_{n}|n+1\rangle\langle n| \sigma_{g e} \mathrm{e}^{i \phi_{n} t}+H . c . .
$$

with $\zeta_{n}=\sqrt{n+1} \zeta$ and $\phi_{n}=(n+1) \xi+\delta-\varpi_{g}-\varpi_{e}$. Next, under the strongly off-resonant regime $\xi \gg \sqrt{k+2}|\zeta|$ and the condition

$$
\phi_{k}=0
$$

which is easily satisfied by imposing $(m+1) \xi=\varpi_{g} \gg \delta=\varpi_{e}$, such that $\left|\Omega_{1}\right|=$ $\sqrt{(m+1) \Delta_{1} / \Delta}|\lambda| \gg \sqrt{\Delta_{1} / \Delta_{2}}\left|\Omega_{2}\right|$, we readily eliminate, via RWA, all the terms proportional to $\zeta_{n}=\sqrt{n+1} \zeta$ summed in $V_{\text {eff }}$, except when $n=k$, bringing about the selective interaction

$$
\mathcal{H}_{1}=\left(\zeta_{k}|k+1\rangle\langle k| \sigma_{g e}+\text { H.c. }\right) \text {, }
$$

producing the desired selective $g \leftrightarrow e$ transition within the Fock subspace $\{|k\rangle,|k+1\rangle\}$. The excellent agreement between this effective selective interaction and the full Hamiltonian (1) has been analyzed in detail in Ref. [17]. Regarding the Hamiltonian associated with the diagram in Fig. 1(b), we readily see that switching off the laser field and tuning the cavity mode to resonance with the atomic transition $g \leftrightarrow i$ results in:

$$
\mathcal{H}_{2}=\tilde{\lambda} \sigma_{i g} a+H . c .
$$

Next, following to the reasoning in Ref. [14, 16] for atomic reservoir engineering, we assume a weak-coupling regime for the interaction parameter associated with $\mathcal{H}_{2}$, i.e., $\tilde{\lambda} \tau \ll$ 1, $\tau$ being the time during which each atom crosses the cavity. However, it is easily verified that the Lindblad structure of the superoperator emerging from $\mathcal{H}_{1}$ does not rely on the weak-coupling regime $\zeta_{k} \tau \ll 1$, owing to the selective nature of this interaction. When the atoms are randomly prepared in the ground, excited, and auxiliary states: $p_{g} \sigma_{g g}+p_{e} \sigma_{e e}+$ $p_{i} \sigma_{i i}$, with the laser detuning $\Delta_{L}$ adjusted to produce $k=m$ and $l$, respectively, we obtain the master equation [14] 


$$
\begin{aligned}
\frac{d \rho}{d t} & =\frac{\gamma_{m}}{2}\left(2 a_{m} \rho a_{m}^{\dagger}-\rho a_{m}^{\dagger} a_{m}-a_{m}^{\dagger} a_{m} \rho\right) \\
& +\frac{\gamma_{l}}{2}\left(2 a_{l}^{\dagger} \rho a_{l}-\rho a_{l} a_{l}^{\dagger}-a_{l} a_{l}^{\dagger} \rho\right) \\
& +\frac{\tilde{\gamma}}{2}\left(2 a^{\dagger} \rho a-\rho a a^{\dagger}-a a^{\dagger} \rho\right)+\mathcal{L} \rho,
\end{aligned}
$$

with the effective rates $\gamma_{m}=r^{g}\left(\zeta_{m} \tau\right)^{2}, \gamma_{l}=r^{e}\left(\zeta_{l} \tau\right)^{2}$, and $\tilde{\gamma}=r^{i}(\tilde{\lambda} \tau)^{2}=\varepsilon \gamma(\varepsilon<1$ to achieve a steady equilibrium state), where $r^{g}, r^{e}$, and $r^{i}$ are the atomic arrival rates proportional to the probabilities $p_{g}, p_{e}$, and $p_{i}$, respectively. The last term in Eq. (7), $\mathcal{L} \rho$, stands for the inevitable Liouvillian operator describing the lossy cavity $(\omega)$ of damping rate $\gamma$ and temperature $T=\hbar \omega / k_{B} \ln [(1+\bar{n}) / \bar{n}], k_{B}$ being the Boltzmann constant, irrespective of the passage of the atoms:

$$
\begin{gathered}
\mathcal{L} \rho=\frac{\gamma}{2}(1+\bar{n})\left(2 a \rho a^{\dagger}-\rho a^{\dagger} a-a^{\dagger} a \rho\right) \\
+\frac{\gamma}{2} \bar{n}\left(2 a^{\dagger} \rho a-\rho a a^{\dagger}-a a^{\dagger} \rho\right) .
\end{gathered}
$$

From the equation of motion for the number state population, $\rho_{n n}=\langle n|\rho| n\rangle$, derived from Eqs. (7) and (8), we obtain the steady state solution (assuming $l+1<m$ ):

$$
\rho_{n n}=\left\{\begin{array}{cc}
R_{n} \rho_{0} & n \leq l \\
R_{n} \mathcal{A}_{l} \rho_{0} & l+1 \leq n \leq m \\
R_{n} \mathcal{B}_{l, m} \rho_{0} & n \geq m+1
\end{array},\right.
$$

where $R_{n}=[(\varepsilon+\bar{n}) /(1+\bar{n})]^{n}$ and

$$
\begin{aligned}
\mathcal{A}_{l} & =\frac{\gamma_{l}+(l+1)(\varepsilon+\bar{n}) \gamma}{(l+1)(\varepsilon+\bar{n}) \gamma}, \\
\mathcal{B}_{l, m} & =\frac{(m+1)(1+\bar{n}) \gamma}{\gamma_{m}+(m+1)(1+\bar{n}) \gamma} \mathcal{A}_{l}, \\
\rho_{0} & =\frac{(1-\varepsilon) /(1+\bar{n})}{1-R_{l+1}+\mathcal{A}_{l}\left(R_{l+1}-R_{m+1}\right)+\mathcal{B}_{l, m} R_{m+1}} .
\end{aligned}
$$

From Eqs. (9) and (10) we clearly see that the distribution function $\rho_{n n}$ can be manipulated by an appropriate choice of the engineered parameters $\gamma_{l}, \gamma_{m}$, and $\tilde{\gamma}$. To estimate the range of validity of these parameters in a microwave cavity QED experiment, we start by choosing $\Delta=\Delta_{1}=\left(1+10^{-2}\right) \times \Delta_{2}=10 \sqrt{k+1}|\lambda|$, such that $\left|\Omega_{1}\right|=10 \times\left|\Omega_{2}\right|=\sqrt{k+1}|\lambda|$, $\zeta_{k}=10^{-2} \sqrt{k+1}|\lambda|, \tau=10^{2} / \sqrt{k+1}|\lambda|$ (so that $\zeta_{k} \tau=1$ [19]). Therefore, assuming 


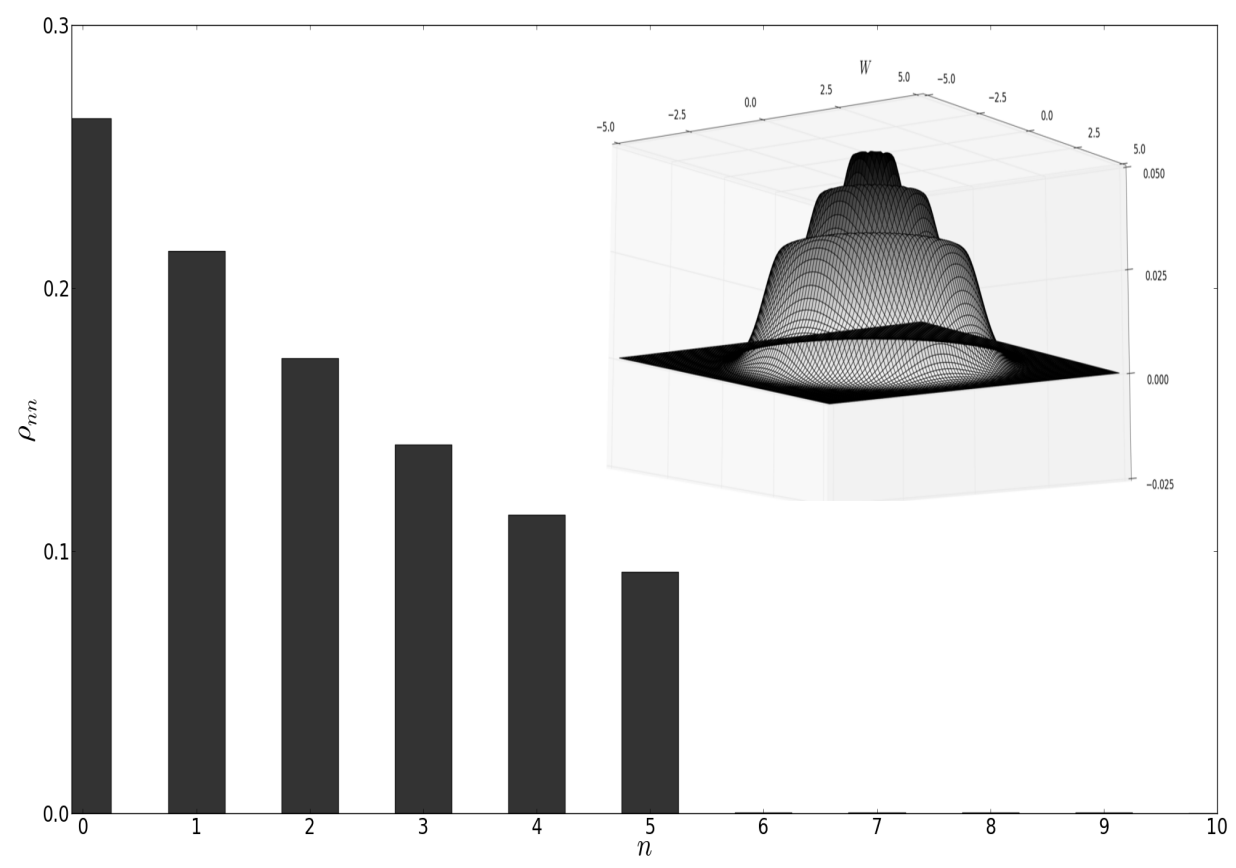

FIG. 2: Truncated thermal distribution from the Fock state $m+1=6$. In the inset we present the Wigner function of the truncated distribution.

$m, l \sim 10$ with typical $\lambda \sim 5 \times 10^{5} \mathrm{~Hz}$ and $\gamma \sim 7.5 \mathrm{~Hz}$, and imposing $\tilde{\gamma} \sim r^{i} \times 10^{-2}=\varepsilon \gamma$, it follows that $\gamma_{k}$ ranges up to the order of $2 \times 10^{3} \gamma$, with $r^{i}=10^{3} \varepsilon$ (i.e., $p_{i}=10^{-2} \varepsilon$ ) and $r^{g}, r^{e}$ ranging up to around $10^{4}\left(p_{g}, p_{e}\right.$ limited to $\left.1-2 p_{i}\right)$.

In order to generate steady Fock states: $i$ ) we first observe that a significantly large $\gamma_{m}$ (relative to $\gamma$ ), with $\gamma_{l}=0$ (no atoms crossing the cavity in the excited state), such that

$$
R_{m+1} \mathcal{B}_{l, m} \ll R_{m} \mathcal{A}_{l}
$$

entails the truncation of the equilibrium distribution $\rho_{n n}$, from the population of state $m+1$, as illustrated in Fig. 2, where we have assumed $m=5$, with $\bar{n}=0.05$ and $\varepsilon=0.8$, such that $\tilde{\gamma}=0.8 \gamma$ and $\gamma_{m}=10^{3} \gamma$ to satisfy Eq. (11). Although the analytical solution in Eqs. (9) and (10) was crucial to alert us to the possibility of manipulating the populations $\rho_{n n}$ through the appropriate choice of the parameters involved, all the simulations in this letter were based on Eq. (7), running in QuTIP [20]. In the inset of Fig. 2, we present the Wigner function of the truncated distribution, which takes no negative values, showing that it is a purely classical state. 


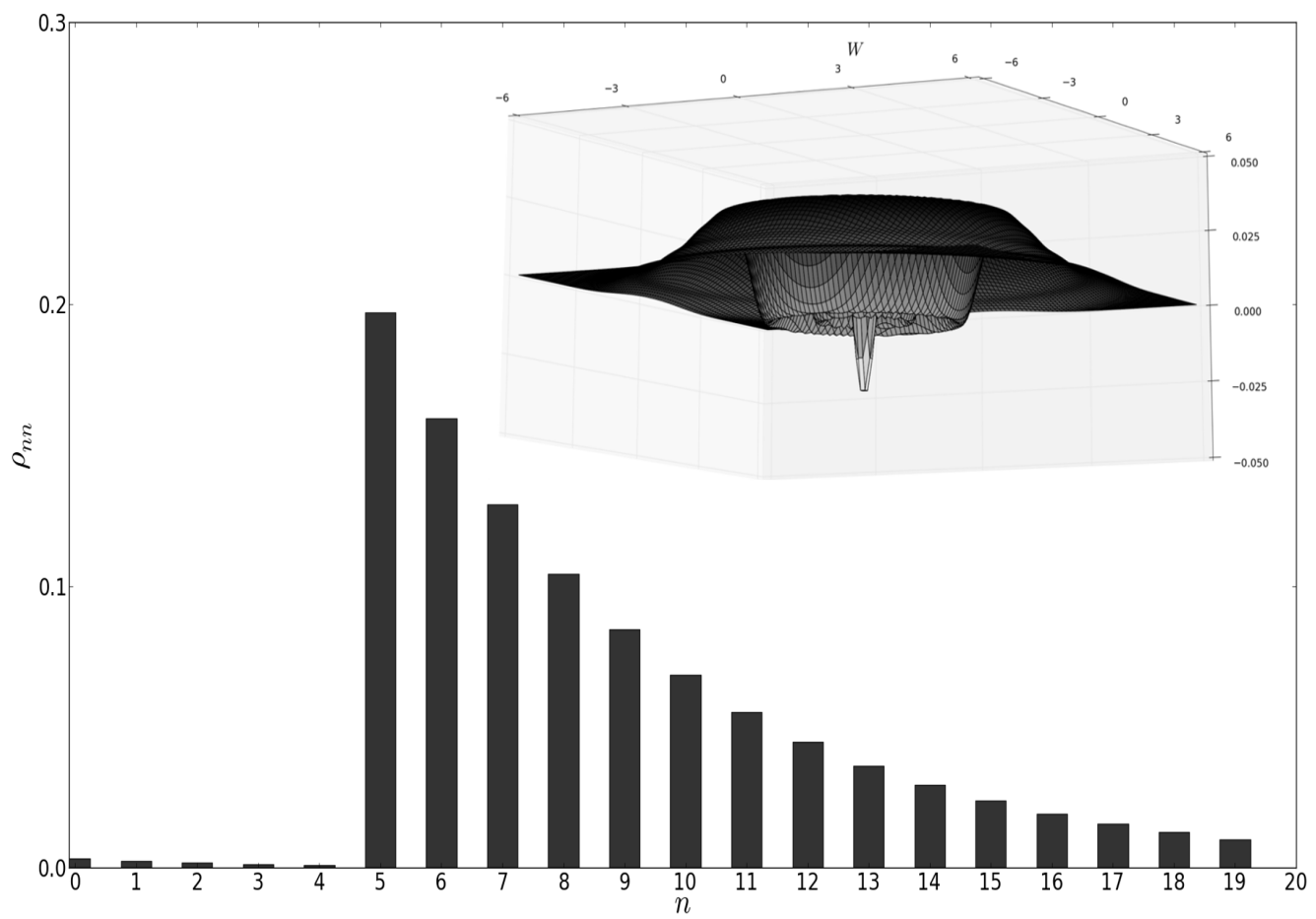

FIG. 3: Amplification of the thermal state distributions, from the Fock state $l+1=5$, with the corresponding Wigner distribution in the inset.

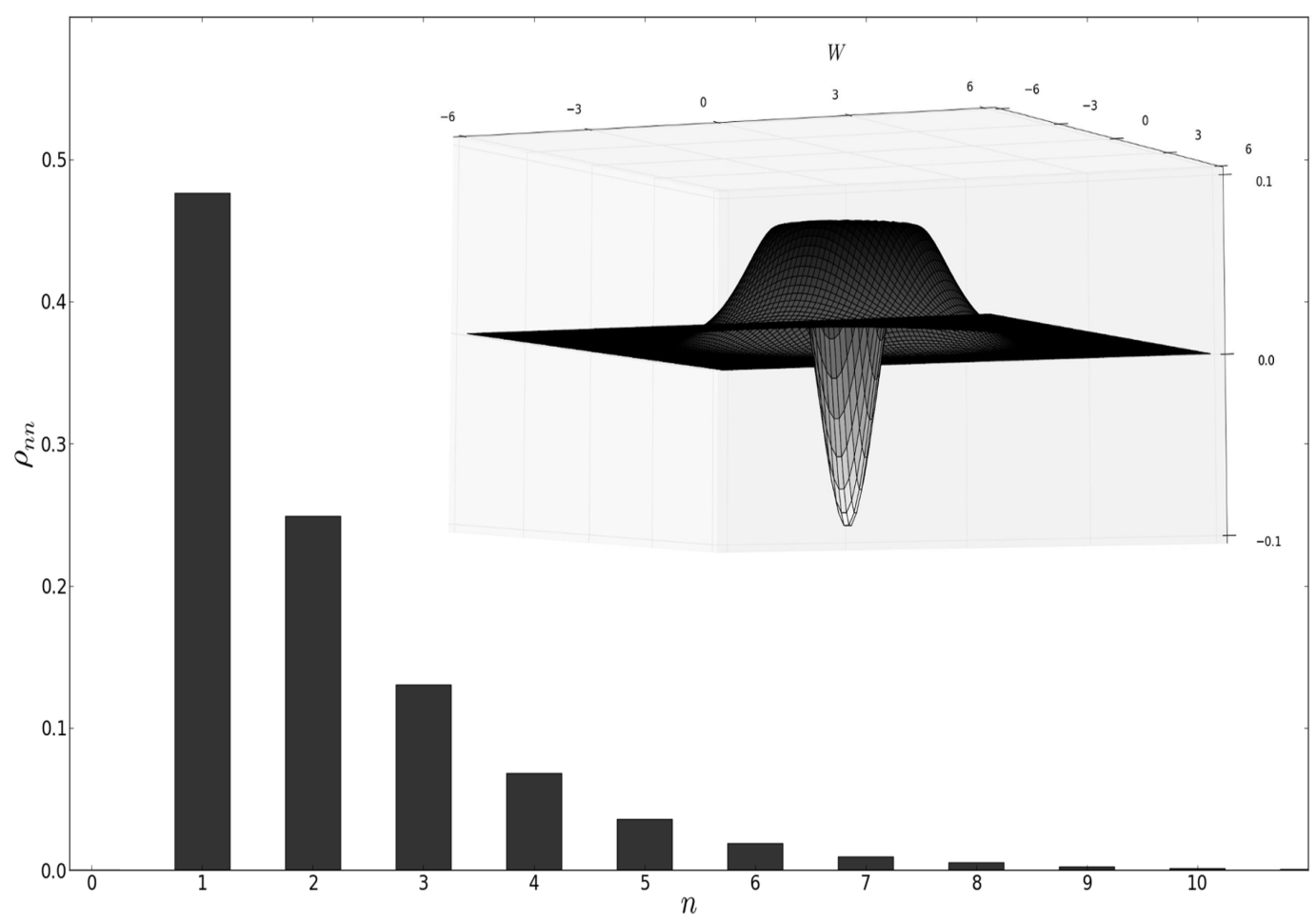

FIG. 4: Amplification of the thermal state distributions, from the Fock state $l+1=1$, with the corresponding Wigner distribution in the inset. 


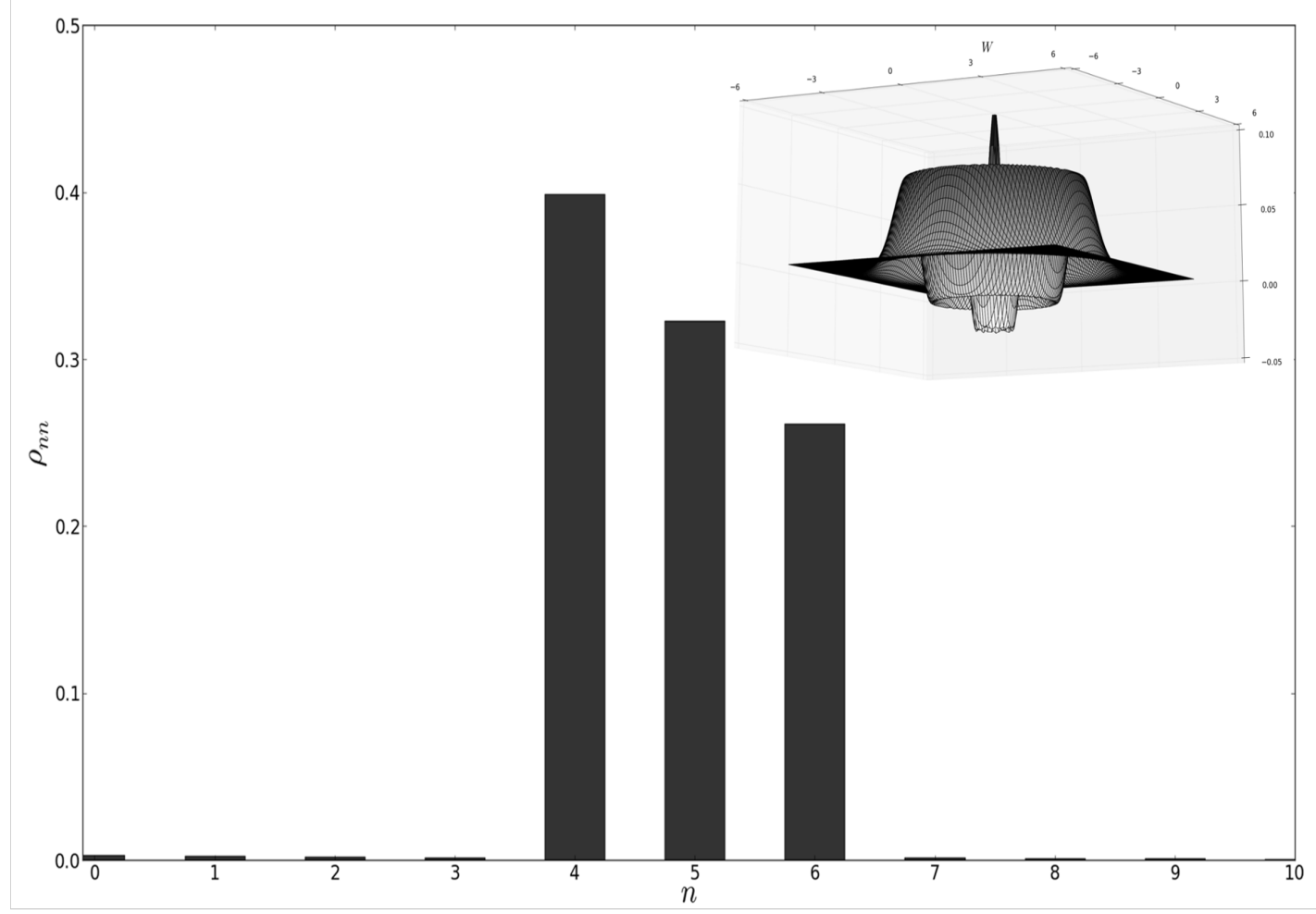

FIG. 5: Sliced thermal distribution, from $l+1=4$ to $m=6$, with the corresponding Wigner distribution in the inset.

ii) On the other hand, a significantly large damping rate $\gamma_{l}$ (relative to $\gamma$ ), with $\gamma_{m}=0$ (no atoms crossing the cavity in the ground state), leads to the effect opposite to that sketched in Fig. 2, enhancing the probabilities $\rho_{n n}$ for $n \geq l+1$, instead of cutting them off. The amplification procedure imposes the restriction

$$
R_{l+1} \mathcal{A}_{l} \gg R_{0}
$$

In Fig. 3, we illustrate the amplification of the equilibrium state, from $l+1=5$, adopting the same parameters as in Fig. 2 but interchanging $\gamma_{m}$ and $\gamma_{l}$, such that $\gamma_{l}=10^{3} \gamma$. The Wigner function in the inset, now exhibiting negative values, shows that this time we obtain a nonclassical state of the radiation field. It is interesting to note, by the way, that by simply eliminating the vacuum state from the thermal distribution, with $l=0$, we automatically obtain a nonclassical state, as shown in Fig. 4, where we use the same parameters as in Fig. 3, but with $\varepsilon=0.5$ (such that $\tilde{\gamma}=0.5 \gamma$ ), to prevent the excitation of large Fock 


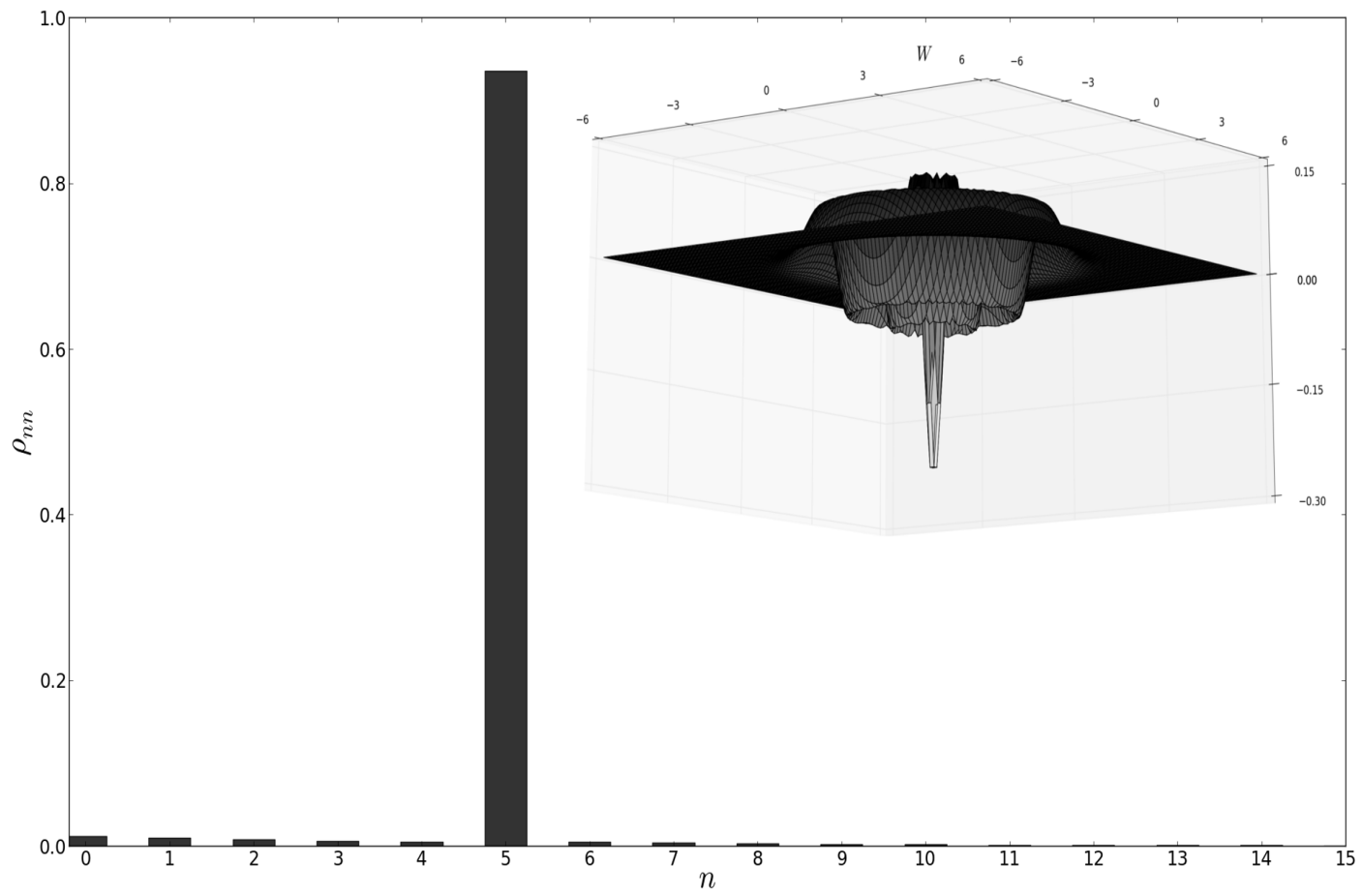

FIG. 6: Steady Fock state $|5\rangle$, with the corresponding Wigner distribution in the inset.

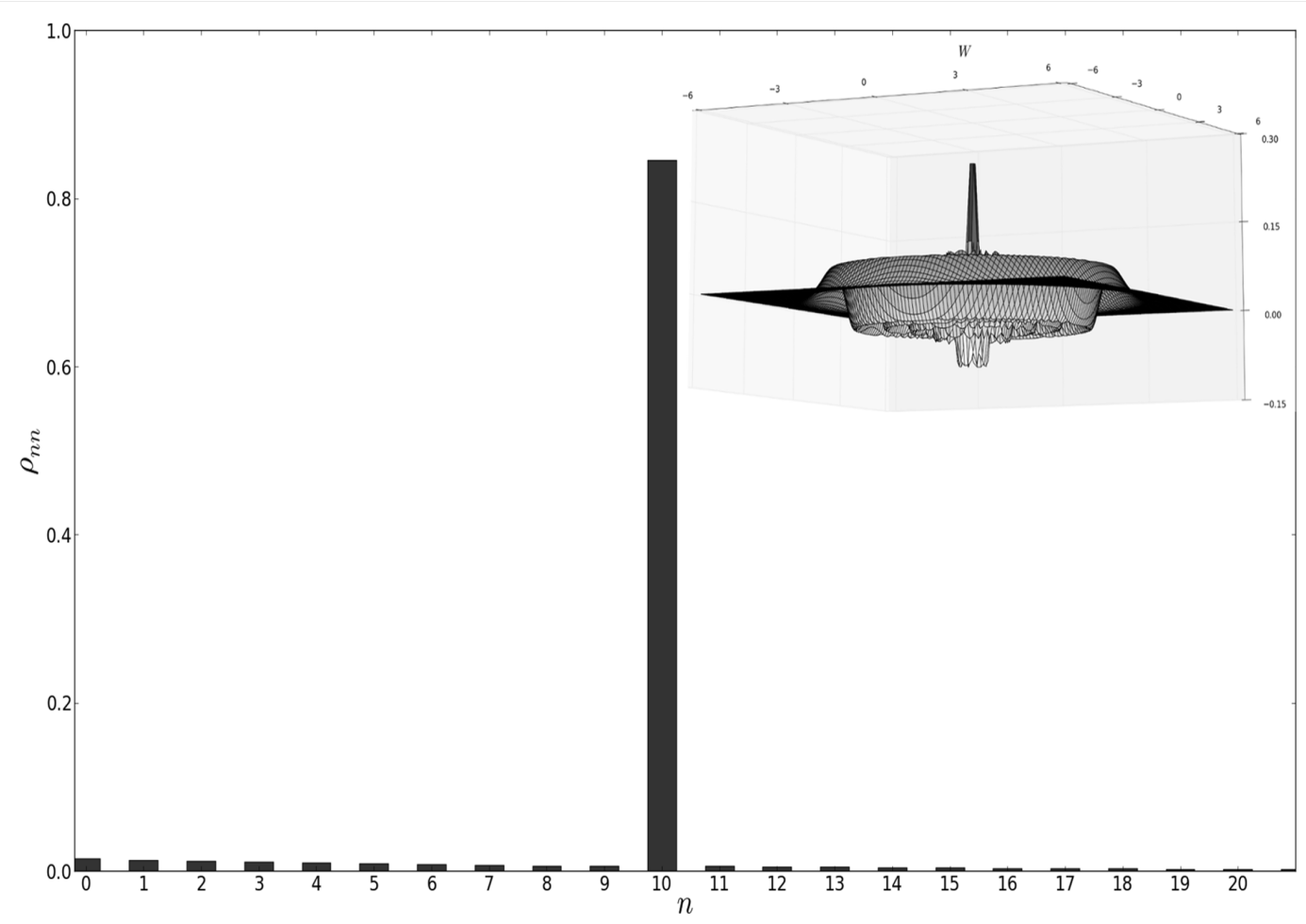

FIG. 7: Steady Fock state $|10\rangle$, with the corresponding Wigner distribution in the inset. 
states. Therefore, the vacuum plays a major role in the intersection between classicality and nonclassicality.

Another case arises when we put together $i$ ) and $i i$ ), so as to $i i i$ ) slice the equilibrium distribution, from $l+1$ to $m$. This is done by ensuring the conditions leading simultaneously to Eqs. (11) and (12). Fig. 5 shows a sliced steady distribution ranging from $l+1=4$ to $m=6$, again assuming the same parameters as above, but $\gamma_{m}=\gamma_{l}=10^{3} \gamma$. The Wigner function in the inset now exhibits a large region with negative values, strengthening the nonclassical character of the generated state. Finally, we come to the main point of our work: the choice $m=l+1$ allows us to join the subspaces $\{l, l+1\}$ and $\{m, m+1\}$ alongside one another, so that both sare the same state $m$. This leads to steady Fock states under the same conditions established in $i i i)$, as seen in Fig. 6, which illustrates the state $m=5$, prepared with exactly the same parameters as in Fig. 5. The fidelity [8] of the prepared Fock state is around 0.97, as confirmed by the Wigner distribution in the inset, which exhibits the peculiar feature of the Fock state. We end up with the Fock state $m=l+1=10$ in Fig. 7, reached with the same parameters in Fig. 4, except $\varepsilon=0.95$, with the fidelity dropped to around 0.88 .

We have thus proposed a scheme to manipulate the steady thermal distribution in such a way as to produce steady Fock states of the radiation field. Our proposal relies on the engineering of selective JC Hamiltonians, which thus generate equally selective Lindblad superoperators that enable us to manipulate the equilibrium thermal distribution, slicing it so as to prepare steady Fock states. Our technique can be implemented in other contexts of atom-field interaction, such as trapped ions and circuit QED, where the beam of atoms simulating the reservoir can be achieved by a pulsed classical field. In the former case, the classical field is used to couple the vibrational field intermittently with the internal ionic states, while in the latter case, it is used to bring a cooper-pair box into resonance with the mode of a superconducting strip. Apart from the preparation of Fock states, other applications within Hamiltonian, reservoir, and state engineering may arise from the present protocol, as for example the generation of entangled steady state in a network of quantum oscillators [21].

\section{Acknowledgements}


The authors acknowledge financial support from PRP/USP within the Research Support Center Initiative (NAP Q-NANO) and FAPESP, CNPQ and CAPES, Brazilian agencies.

[1] For the engineering schemes relying on atomi-state measurement, see K. Vogel, V. M. Akulin, and W. P. Schleich, Phys. Rev. Lett. 71, 1816 (1993); R.M. Serra, N. G. de Almeida, C. J. Villas-Boas, and M. H. Y. Moussa, Phys. Rev. A 62, 043810 (2000); and for those not requiring atomi detection, see A. S. Parkins, P. Marte, P. Zoller, and H. J. Kimble, Phys. Rev. Lett. 71, 3095 (1993); Th. Wellens, A. Buchleitner, B. Kümmerer, and H. Maassen, Phys. Rev. Lett. 85, 3361 (2000).

[2] M. A. de Ponte, S. S. Mizrahi and M. H. Y. Moussa, Phys. Rev. A 84, 012331 (2011); D. A. Lidar, I. L. Chuang, and K. B. Whaley, Phys. Rev. Lett. 81, 2594 (1998).

[3] L. Viola and E. Knill, Phys. Rev. Lett. 94, 060502 (2005); L. C. Celeri, M. A. de Ponte, C. J. Villas-Boas, and M. H. Y. Moussa, J. Phys. B 41, 085504 (2008).

[4] J. F. Poyatos, J. I. Cirac, and P. Zoller, Phys. Rev. Lett. 77, 4728 (1996).

[5] F. O. Prado, E. I. Duzzioni, M. H. Y. Moussa, N. G. de Almeida, and C. J. Villas-Bôas, Phys. Rev. Lett. 102, 073008 (2009).

[6] M. Brune, E. Hagley, J. Dreyer, X. Maître, A. Maali, C. Wunderlich, J. M. Raimond, and S. Haroche; Phys. Rev. Lett. 77, 4887-4890 (1996)

[7] M. Brune, J. Bernu, C. Guerlin, S. Dele'glise, C. Sayrin, S. Gleyzes, S. Kuhr, I. Dotsenko, J.-M. Raimond, and S. Haroche, Phys. Rev. Lett. 101, 240402 (2008).

[8] M. Nielsen, I. Chuang, Quantum Computation and Quantum Information, Cambridge University Press, 2000, 409-416.

[9] C. J. Myatt, B. E. King, Q. A. Turchette, C. A. Sackett. D. Kielpinski, W. M. Itano, C. Monroe, and D. J. Wineland, Nature 403, 269 (2000).

[10] X. Zhou, I. Dotsenko, B. Peaudecerf, T. Rybarczyk, C. Sayrin, S. Gleyzes, J. M. Raimond, M. Brune, and S. Haroche, Phys. Rev. Lett. 108, 243602 (2012).

[11] B. T. H. Varcoe, S. Brattke, M. Weidinger, and H. Walther, Nature 403, 743 (2000); P. Bertet, S. Osnaghi, P. Milman, A. Auffeves, P. Maioli, M. Brune, J. M. Raimond, and S. Haroche, Phys. Rev. Lett. 88, 143601 (2002).

[12] D. M. Meekhof, C. Monroe, B. E. King, W. M. Itano, and D. J. Wineland, Phys. Rev. Lett. 
76, 1796 (1996); D. Leibfried, D. M. Meekhof, B. E. King, C. Monroe, W. M. Itano, and D. J. Wineland, Phys. Rev. Lett. 77, 4281 (1996).

[13] M. Hofheinz, E. M. Weig, M. Ansmann, R. C. Bialczak1, E. Lucero, M. Neeley, A. D. O’Connell, H. Wang, J. M. Martinis, and A. N. Cleland, Nature 454, 310 (2008).

[14] B.-G. Englert and G. Morigi, in Coherent Evolution in Noisy Environments, edited by A. Buchleitner and K. Hornberger (Springer, Berlin, 2002), p. 55.

[15] J. M. Raimond, M. Brune, and S. Haroche, Rev. Mod. Phys. 73, 565 (2001).

[16] S. Pielawa, G. Morigi, D. Vitali, and L. Davidovich, Phys. Rev. Lett. 98, 240401 (2007); S. Pielawa, L. Davidovich, D. Vitali, and G. Morigi, Phys. Rev. A 81, 043802 (2010); S. Pielawa, G. Morigi, D. Vitali, and L. Davidovich, Phys. Rev. A 85, 022120 (2012).

[17] F. O. Prado, W. Rosado, A. M. Alcalde, and M. H. Y. Moussa, J. Phys. B: At. Mol. Opt. Phys. 46, 205501 (2013).

[18] O. Gamel and D. F. V. James, Phys. Rev. A 82, 052106 (2010); D. F. V. James and J. Jerke, Can. J. Phys. 85, 625 (2007).

[19] We performed (following the reasoning in Refs. [14, 16]) a numerical simulation of the passage of atoms through the cavity, assuming $\tau=10^{-4} \mathrm{~s}$ in conformity with $\zeta_{k} \tau=1$, to confirm that the cavity mode evolves in excellent agreement with the coarse-graining master equation (7), and that higher value of $\tau$ start to compromise the accuracy of this master equation.

[20] J. R. Johansson, P. D. Nation, and F. Nori, Comput. Phys. Commun. 183, 1760 (2012); ibid. 184, 1234 (2013).

[21] G. D. de Moraes Neto et al., to be published elsewhere. 